\title{
The Critical Role of Health Information Technology in the Safe Integration of Behavioral Health and Primary Care to Improve Patient Care
}

\author{
Mark Segal, PhD, FHIMSS \\ Patricia Giuffrida ${ }^{\oplus}$, MSN, RN, CPHIMS ECRI \\ Lorraine Possanza, DPM, JD, MBE, FACFOAM, FAPWCA, ECRI \\ David Bucciferro, BS, Certification in Psychiatric Rehabilitation
}

\begin{abstract}
Integrated behavioral health and primary care is a set of related approaches to enhance care delivery, safety, and quality through closer collaboration and coordination among clinicians and care organizations in these two fields. Technology (e.g., electronic health records (EHR), telehealth, clinical decision support, and standards-based interoperability) can enable integration and improve delivery of care. Reinforcing these opportunities, virtual care and telehealth have dramatically changed care availability and delivery, especially during the COVID-19 pandemic. There are different levels of integration, ranging from minimal to full collaboration. The Partnership for Health IT Patient Safety and the HIMSS Electronic Health Record Association (EHRA) formed a workgroup to examine using information technology to facilitate integration. Taking a three-pronged approach focused on (1) screening for behavioral health issues, (2) clinician documentation, and (3) sharing data among clinicians, patients, and authorized parties, the workgroup developed action-oriented recommendations and strategies for safe use of health IT for stakeholders and policymakers seeking to advance efforts to integrate behavioral health with primary care.
\end{abstract}

Address correspondence to Patricia Giuffrida, MSN, RN, CPHIMS ECRI, Plymouth Meeting, Montgomery, PA, USA. pgiuffrida@ecri.org.

Lorraine Possanza, DPM, JD, MBE, FACFOAM, FAPWCA, ECRI, Plymouth Meeting, Montgomery, PA, USA

Mark Segal, PhD, FHIMSS, Digital Health Policy Advisors, LLC, Cook County, IL, USA.

David Bucciferro, BS, Certification in Psychiatric Rehabilitation, Foothold Technology, New York, NY, USA.

The Journal of Behavioral Health Services \& Research, 2022. 221-230. (C 2021, National Council for Mental Wellbeing. DOI 10.1007/s11414-021-09774-0 


\section{Introduction}

Integrated behavioral health and primary care (integration) is an effective set of related approaches to enhancing patient care delivery, safety, and quality through closer collaboration and coordination among clinicians and care organizations in these two fields. ${ }^{1,2}$ For the purposes of this paper, behavioral health is defined as "an umbrella term that includes mental health and substance abuse conditions, life stressors and crises, stress-related physical symptoms, and health behaviors."1 (P.1)

Technology tools, including electronic health records (EHRs), telehealth, clinical decision support (CDS), clinical registries, quality measure dashboards, and standards-based interoperability, can enable integration among various clinical disciplines and more generally improve the delivery of care so that it more readily addresses patient needs. ${ }^{3}$ Reinforcing these opportunities, virtual care and telehealth have dramatically changed care availability and delivery, prior to and especially during the COVID-19 pandemic, providing a firm base for using these tools to further enhance integration. ${ }^{4-6}$

These technology-enabled changes have been and will continue to be facilitated by new and revised regulations addressing telehealth, patients' access to their electronic data, interoperability, and information blocking. ${ }^{7-9}$ These regulations seek to break down traditional silos of care and data and to increase the quality, quantity, and completeness of standardized clinical data, enabling clinical information to become more widely available and useful to those who need it. These regulations and associated policy initiatives also are intended to enable access and exchange of large quantities of high-quality structured and unstructured data that can support predictive analytics and clinical decision support and other technology-enabled approaches that will benefit from artificial intelligence, especially machine learning. ${ }^{10}$

There are different levels of integration of primary care and behavioral health. ${ }^{11}$ Each uses different approaches, ranging from minimal to full collaboration among behavioral health and primary care clinicians in a transformed or merged practice. Although the integration model used does not alter the ability of technology to facilitate integration, it can affect clinical and administrative mechanisms and operations and opportunities offered by specific technologies. The integration models to be supported will need to be considered in decisions in development and especially implementation of technology by developers and healthcare organizations.

During 2020, the Partnership for Health IT Patient Safety and EHRA formed a workgroup to look closely at using information technology to facilitate integration. ${ }^{12,13}$ Taking a three-pronged approach focused on (1) screening for behavioral health issues, (2) clinician documentation, and (3) sharing data among clinicians, patients, and authorized parties, the workgroup developed recommendations and strategies for safe use of health IT for integrating these disciplines.

These three interrelated categories of action can differ in their execution in behavioral health and in primary care. To effectively improve care, information must be captured, with vocabularies (concepts and terms used by behavioral health and primary care clinicians as well as terminologies used to represent clinical data) understood by those who both create and access clinical data, and then must be transferred and shared among clinicians and other users. Importantly, tools must be available to assist in the recognition of clinical needs so care can be initiated, communicated, measured, and shared.

As part of its work, the group used the workflow algorithm in Figure 1, which identifies areas for application of health IT to facilitate integration. This workflow follows a patient's visit through care options and highlights where technology can facilitate screening, documentation, and sharing of information.

At the conclusion of its project, the group provided action-oriented safe practice recommendations for EHR and health IT developers, clinicians and healthcare organizations, the government, and other stakeholders to facilitate their efforts to implement and incorporate behavioral health with primary care. 


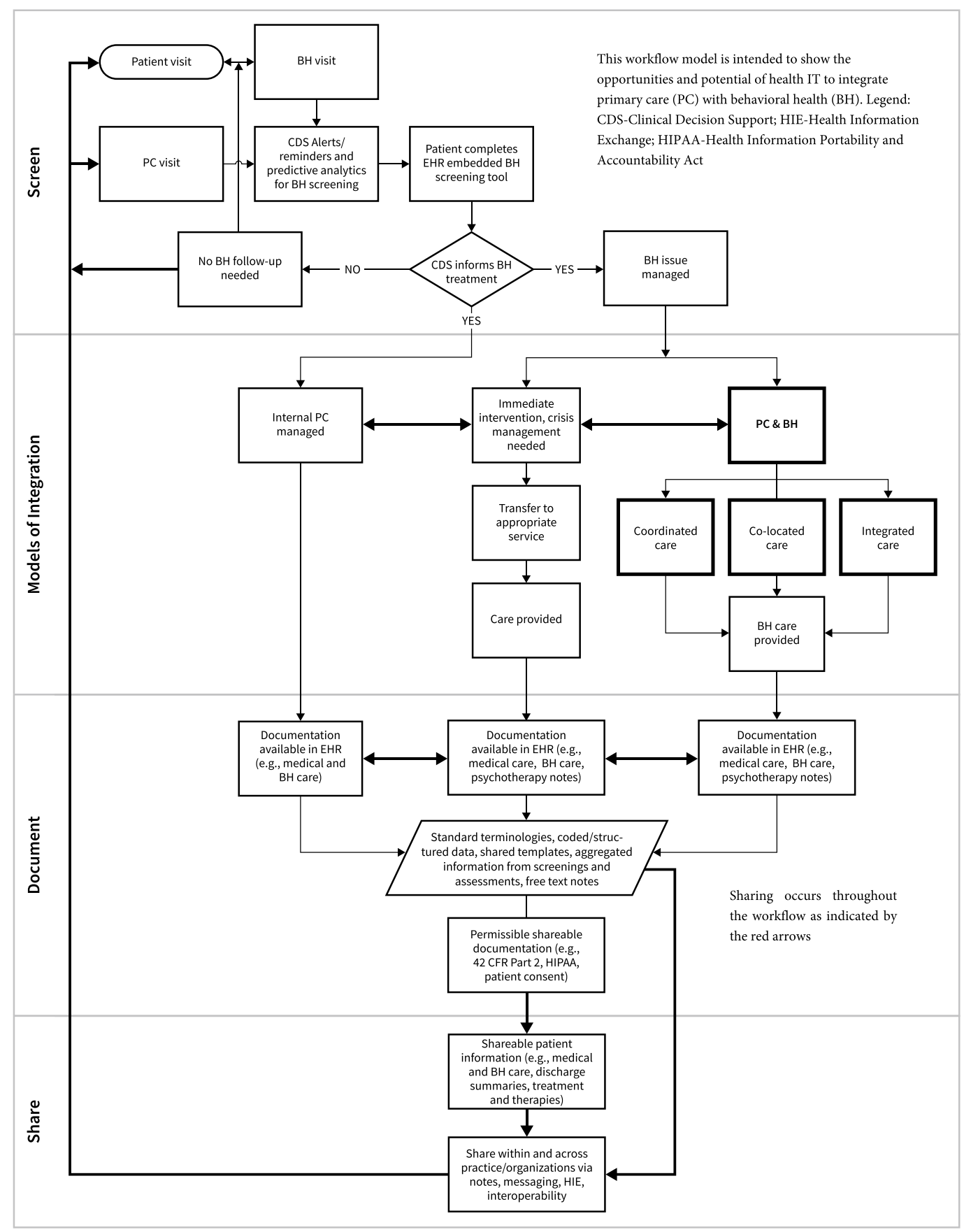

\section{Figure 1}

IT-enabled workflow for the safe integration of primary care and behavioral health. Source: Partnership for Health IT Patient Safety. Optimizing health IT for safe integration of behavioral health and primary care [white paper]. Plymouth Meeting (PA): ECRI; 2021. 48 p. Also available: https://www.ecri.org/solutions/hit-partnership 


\section{Methods}

EHRA, an association of EHR developers, recruited a workgroup from its members that ultimately included representatives from 15 developer companies. All member companies were invited to provide representatives, and neither the Partnership for Health IT Patient Safety or EHRA limited or influenced workgroup composition. The resulting 21 developer workgroup members (11 men and 10 women) were from a mix of smaller, mid-size, and larger organizations serving both ambulatory and inpatient-oriented providers and clinicians. Members were a mix of technical experts and clinicians, including individuals with direct experience in primary care and behavioral health settings.

Partnership for Health IT Patient Safety coordinated and led workgroup efforts. The fundamental model of the Partnership for Health IT Patient Safety workgroup activities is a proactive learning system, using an approach that is neither punitive nor regulatory. Partnership for Health IT Patient Safety and EHRA began by investigating and identifying the health IT-related issues that can determine the feasibility and effectiveness of integration of behavioral health and primary care. Virtual meetings were held over 9 months. The workgroup followed the Partnership for Health IT Patient Safety workgroup issue qualitative evaluation process, which includes review of ECRI and the Institute for Safe Medication Practices (ISMP) Patient Safety Organization (an organization that collects and analyzes data voluntarily reported by healthcare providers to help improve patient safety and healthcare quality, operating under the Patient Safety and Quality Improvement Act of $2005)^{14}$ data, evidence-based literature reviews, and most importantly, workgroup deliberations to identify areas of focus and specific recommendations that emerged from workgroup discussions and informal consensus. ${ }^{15}$ Meetings included a mix of formal presentations, group discussions, online collaboration, and expert speakers. The information and learnings obtained from these various sources were used to develop safe practice recommendations and suggest strategies to execute them.

\section{Recommendations and Rationale}

\section{Overview}

Recommendations on screening, documentation, and sharing were developed and refined through a focus on functionality, data elements and fields, integration, and measures. The recommendations reflect current technology and regulations and are intended to create awareness among key stakeholders and policymakers and provide a framework for near-term and future action. ${ }^{16}$

Although needed technologies are increasingly available, they are not universally implemented or adopted in either behavioral health or primary care EHRs. Moreover, behavioral health and primary care clinicians often document information in varying ways (e.g., a summary behavioral health clinical or case note vs. structured data in a primary care EHR that records discrete data elements such as disorder, episode, course, and severity $)^{17}$ and with different professional vocabularies (concepts and terms used by behavioral health and primary care clinicians in clinical notes and similar communications), capturing information in different fields or describing the same data using diverse clinical terminologies (e.g., DSM-5, ICD-10, or CPT). Sharing between behavioral health and primary care is also often limited by available technology (e.g., whether clinicians have an EHR in addition to their billing system, work in the same EHR, have the capability to generate or receive structured clinical documents such as the $\mathrm{C}-\mathrm{CDA}^{18}$, or have health information exchange capabilities) as well as regulations such as HIPAA and 42 CFR Part 2 and concerns about whether protected information will remain protected once shared.

The recommendations were developed to apply to all levels and models of integrated care, although organizational and technical approaches may vary. The workgroup also identified potential 
measures to assess progress toward integration, including care and quality measures. Developers and clinicians must work together to ensure that needed information is captured to support such measures. Each of the three areas—screening, documentation, and sharing-includes specific recommendations: ${ }^{16}$

\section{Screening}

\section{Ensure that validated, clinically accepted screening tools are integrated, easily accessible, and readily available in the EHR}

Streamlining screening in both primary care and behavioral health settings, using validated, standardized tools, can increase identification of patients needing behavioral health services. ${ }^{19-21}$ Screening tools should be integrated in or available through the EHR, including in patient portals, and able to display usable results for clinicians and healthcare organizations, facilitate communication of screening results between clinicians to enhance care coordination, and support internal and external reporting.

\section{Enable triggers for clinical decision support (CDS) associated with screening tools to integrate behavioral health and primary care}

CDS can be used effectively in primary care settings to facilitate screening for behavioral health issues, including depression and substance use disorder. ${ }^{22-24}$ CDS can also inform follow-up recommendations based on screening results. EHRs should enable "triggers" that initiate use of CDS tools associated with commonly used screening tools based on specific clinical data (e.g., using standards like CDS Hooks, which invoke decision support from within a clinician's workflow) and support data elements needed to support such triggers. ${ }^{25}$ Links between screening tools and CDS help to streamline workflow, implement evidence-based care, and drive opportunities for predictive analytics.

\section{Documentation}

\section{Optimize documentation to support integration of behavioral health and primary care}

To appropriately diagnose, treat, and track care, clinicians communicate through clinical documentation. It is widely understood that EHR documentation needs to be simplified and standardized to support care, communications with other clinicians and patients, data extraction, and clinical performance reporting. ${ }^{26}$ Although clinicians may differ in how they document or, depending on specialty, what is documented, documentation is essential to clinical care and to enable communication via shared documentation to realize benefits of integrated care. Strategies to optimize documentation include streamlining behavioral health data entry, ensuring accurate representation of data, directly or via mapping, and making needed data elements available in health IT in a standardized form.

The workgroup also considered current and future states of the US Core Data for Interoperability (USCDI) developed by the US Office of the National Coordinator for Health IT, including new data elements for social determinants of health (SDOH). ${ }^{27,28}$ There is extensive work underway to standardize terminology for SDOH, including ICD-10-CM "Z Codes" and the Gravity Project. ${ }^{29,30}$ The workgroup recognized that the ability of technology to incorporate specific data elements is at different stages of development based on variable maturity of standards. ${ }^{31}$ The implementation of existing standards for tagging and segmenting data elements is also needed to assist in compliance with regulatory requirements for data relating to confidentiality of substance use disorder patient records (i.e., 42 CFR Part 2) while also enabling effective sharing between clinicians. ${ }^{32,33}$ 


\section{Sharing}

Enable information sharing across care environments (within organizations, among clinicians in the same system, or across organizations and systems) and with portals, secure messaging, and health information exchange (HIE).

Depending on the integration model, information may be shared primarily within or across systems. Technical considerations in each model or setting can define the mechanics of how regulations are implemented as well as how technologies are designed and implemented. These sharing capabilities should be added by developers and implemented by care delivery organizations. Data sharing capabilities should include granular (i.e., data element-level) clinical information to support various scenarios and requirements, including:

- Sharing within and across healthcare systems between healthcare organizations and clinicians

- Segregating information to selected clinicians and other authorized parties in compliance with the requirements of 42 CFR Part 2 (using structured and encoded data) ${ }^{33}$

- Standards-based interoperability and information exchange, including support for document-level (e.g., HL7® C-CDA) and granular data (e.g., HL7® FHIR®) standards ${ }^{18,34}$

- Support additional uses like billing and pre-authorizations

- Quality measurement and other reporting (e.g., public health reporting)

- Sharing with patients and caregivers

Enable EHRs to use information in the record to segment patient information for exchange consistent with organizational policies, patient requests, and state and federal laws and regulations.

Despite the clinical and safety benefits of sharing to facilitate integration, access by other clinicians to behavioral health documentation can be restricted by patient preferences, organizational policies and practices, federal and state laws, and technology configurations reflecting these policies and understanding of such laws. To achieve the sharing needed for integration, barriers to data access should be intentional, well understood, and no more than needed to ensure regulatory compliance and respect patient preferences. EHRs should include data segmentation capabilities to support HIPAA, 42 CFR Part 2, and patient consent, including ONC certification criteria for sending and receiving data. ${ }^{31,35-37}$ It is also essential that clinicians have the tools, training, and workflows to effectively use segmentation capabilities.

\section{Implications for Behavioral Health}

Developers, healthcare organizations, and clinicians face many challenges and uncertainties because of the COVID-19 pandemic and associated increases in demand for behavioral health services, new regulations, limited resources, and an increase in telehealth visits. ${ }^{4,38,39}$ In the face of these pressures, as well as pre-existing opportunities to control healthcare costs, address comorbidities more effectively, and increase care coordination, the need to integrate behavioral health with primary care is increasingly pressing. Moreover, the rapid and continuing shift of behavioral health visits to telehealth with COVID-19 (e.g., $56 \%$ of all behavioral health visits used telehealth in one cross-specialty study as of December 2020, the highest of any specialty $)^{40}$ illustrates the extent to which behavioral health has changed because of and can be enhanced by technology tools. ${ }^{4}$

\section{Developers}

Health IT has a critical role to play in facilitating safe integration. and optimizing health IT for safe integration is a shared responsibility. Implementing the workgroup recommendations will lay a foundation for interoperable optimization of integrated behavioral health with primary care by: 
- Designing, deploying, and implementing health IT to optimize integration

- Enabling bidirectional communication for behavioral health information within and across organizations

- Applying user design concepts

- Incorporating data segmentation capabilities

At the same time, as developers are being challenged to optimize technology, clinicians, regulators, and patients must also embrace their roles in enabling and improving integrated care by recognizing the importance of such models and supporting their growth and refinement through research, funding, public policy, and patient advocacy.

\section{Clinicians and Healthcare Organizations}

The collaboration of clinicians and healthcare organization with developers can improve the integration of behavioral health and primary care by:

- Selecting and implementing validated behavioral health screening tools specific to the patient population served

- Creating smooth workflows that align with the electronic workflow to simplify the screening process for behavioral health issues

- Ensuring that available functionality for integration is used to its full potential

- Understanding unmet functionality and process needs of the EHR

- Leveraging APIs to record and communicate the results of the embedded screening tools

- Participating in priority policy and regulatory efforts to further the integration of behavioral health and primary care

\section{Policy}

Developers, clinicians, and healthcare organizations should support and encourage regulatory efforts to integrate behavioral health and primary care by:

- Minimizing regulatory documentation requirements

- Driving efforts to enhance health information infrastructure to support interoperability, usability, and information exchange

- Requesting the congress and HHS to establish a legal and regulatory framework to enable the safe sharing of behavioral health data

Notably, the proposed rules released in December 2020 from the Centers for Medicare and Medicaid Services ${ }^{41}$ (CMS) and the Department of Health and Human Services Office of Civil Rights (OCR) included requests for information on data segmentation, ${ }^{41}$ how to advance electronic data exchange among behavioral health providers ${ }^{30}$ and encouraging disclosures of protected health information to help persons experiencing substance use disorder and serious mental illness. ${ }^{42}$ The US federal government policy changes in these areas, in addition to recent policy changes affecting interoperability and data access ${ }^{7-9}$, could enable greater inter-clinician access to and use of patients' health data in ways that enhance integration and resulting improvements in clinical care while protecting patient's privacy and respecting their wishes for use of their health data.

The recommendations reported on in this paper build on one another as data captured from screening is added to documentation and shared within and across healthcare organizations and with 
patients. Health IT can facilitate information exchange regardless of which clinician is providing care and plays a role in integrated care regardless of the setting or the level of integration. ${ }^{16}$

The positive benefits ${ }^{18,43,44}$ for behavioral health of such an approach are.

- Improved communications and relationships among clinicians and their patients

- Enhanced continuity of care

- Better care for patients

- More complete reflection of patient preferences for information sharing

Further research is needed to determine if integrating behavioral health and primary care using embedded screening tools, improved documentation, and exchange of patient information using health information technology will improve patient clinical outcomes. Researchers are in a position to provide the evidence developers, clinicians, and healthcare organization need to further the integration of behavioral health and primary care using health information technology.

Acknowledgements ECRI and the Gordon and Betty Moore Foundation supported this work, with in-kind support from the HIMSS Electronic Health Record Association (EHRA), and the authors thanks these organizations for their generous support in building a sustainable private sector infrastructure for effective health IT patient safety practices. The content is solely the responsibility of the authors and does not necessarily represent the official views of ECRI, the Gordon and Betty Moore Foundation, or the EHRA.

The authors thank all of those who contributed to the workgroup and the development of safe practices. They also thank those who presented to the workgroup throughout its meetings, in particular Kristen Lambert, JD, MSW, LICSW, FASHRM, CPHRM (Trust Risk Management Services); Evelyn A. Segal, PhD (Loyola University), Associate Professor of Family Medicine and Psychiatry \& Behavioral Neurosciences; and Hans J. Buitendijk, MSc, FHL7 (Cerner), Director Interoperability Strategy and Chair of the EHRA. We are also grateful for the assistance provided by EHRA staff: Kristi Feliksik; Jessie Bird, CAE; and Kasey Nicholoff.

\section{Declarations}

Conflict of Interest This work was supported in part by ECRI and the Gordon and Betty Moore Foundation, with in-kind support from the HIMSS Electronic Health Record Association (EHRA). The content is solely the responsibility of the authors and does not necessarily represent the official views of ECRI or the Gordon and Betty Moore Foundation.

\section{References}

1. The Academy for Integrating Behavioral H, Primary C. What is integrated behavioral health? 2020; https://integrationacademy.ahrq. gov/about/integrated-behavioral-health. Accessed 11 May 2021.

2. Malâtre-Lansac A, Engel CC, Xenakis L, et al. Factors influencing physician practices' adoption of behavioral health integration in the United States: a qualitative study. Annals of Internal Medicine. 2020;173(2):92-99.

3. Institute of M, Committee on Quality of Health Care in A. Crossing the quality chasm: a new health system for the 21st century. Washington (DC): National Academies Press; 2001.

4. 4.Pierce BS, Perrin PB, Tyler CM, et al. The COVID-19 telepsychology revolution: a national study of pandemic-based changes in U.S. mental health care delivery. American Psychology Association. 2020;76(1):14-25.

5. Townley C, Yalowich R. Improving behavioral health access \& integration using telehealth \& teleconsultation: a health care system for the 21st century. Portland (ME): National Academy for State Health Policy (NASHP); 2015.

6. Barnett ML, Huskamp HA. Telemedicine for mental health in the United States: making progress, still a long way to go. Psychiatric services (Washington, DC). 2020;71(2):197-198.

7. Final policy, payment, and quality provisions changes to the Medicare physician fee schedule for calendar year 2021. 2020; https://www. $\mathrm{cms}$.gov/newsroom/fact-sheets/final-policy-payment-and-quality-provisions-changes-medicare-physician-fee-schedule-calendar-year- 1 . Accessed 8 Dec 2020. 
8. Interoperability and patient access fact sheet. 2020; https://www.cms.gov/newsroom/fact-sheets/interoperability-and-patient-accessfact-sheet. Accessed 8 Dec 2020.

9. ONC's Cures Act Final Rule. https://www.healthit.gov/curesrule/. Accessed 8 Dec 2020.

10. Arbet J, Brokamp C, Meinzen-Derr J, et al. Lessons and tips for designing a machine learning study using EHR data. Journal for Clinical Translational Sci. 2020;5(1):e21.

11. Heath B, Romero P, Reynolds K. A standard framework for levels of integrated healthcare. Washington (DC): SAMHSA-HRSA Center for Integrated Health Solutions; 2013.

12. Partnership for Health IT Patient Safety. https://www.ecri.org/solutions/patient-safety-organization. Accessed 8 Dec 2020.

13. HIMSS Electronic Health Record Association (EHRA). https://www.ehra.org. Accessed 8 Dec 2020.

14. Patient Safety Organization (PSO) program. https://pso.ahrq.gov/. Accessed 12 May 2021.

15. ECRI and the Institute for Safe Medication Practices PSO. https://www.ecri.org/solutions/patient-safety-organization. Accessed 8 Dec 2020.

16. Partnership for Health ITPS. Optimizing health IT for safe integration of behavioral health and primary care [white paper]. Plymouth Meeting (PA): ECRI; 2021.

17. Ranallo PA, Kilbourne AM, Whatley AS, et al. Behavioral health information technology: from chaos to clarity. Health Affairs. 2016;35(6):1106-1113.

18. C-CDA (HL7 CDA® R2 Implementation Guide: Consolidated CDA Templates for Clinical Notes - US Realm). 2019; http://www. hl7.org/implement/standards/product_brief.cfm?product_id=492. Accessed 10 May 2021.

19. 19.Frick MG, Butler SA, deBoer DS. Universal suicide screening in college primary care. Journal of American College Health. 2019;1:1-6.

20. Sudhanthar S, Thakur K, Sigal Y, Turner J. Improving validated depression screen among adolescent population in primary care practice using electronic health records (EHR). BMJ Quality Improvement Reports. 2015;4(1):u209517.

21. Bajracharya P, Summers L, Amatya AK, et al. Implementation of a depression screening protocol and tools to improve screening for depression in patients with diabetes in the primary care setting. The Journal for Nurse Practitioners. 2016;12(10):690-697.

22. Burdick TE, Kessler RS. Development and use of a clinical decision support tool for behavioral health screening in primary care clinics. Applied Clinical Informatics Journal. 2017;8(2):412-429.

23. Dela Cruz AM, Walker R, Pipes R, et al. Creation of an algorithm for clinical decision support for treatment of opioid use disorder with buprenorphine in primary care. Addict Science and Clinical Practice. 2021;16(1):12.

24. 24.Price-Haywood EG, Robinson W, Harden-Barrios J, et al Intelligent clinical decision support to improve safe opioid management of chronic noncancer pain in primary care. The Ochsner Journal. 2018;18(1):30-35.

25. CDS hooks: overview. https://cds-hooks.org/. Accessed 8 Dec 2020.

26. Payne TH, Corley S, Cullen TA, et al. Report of the AMIA EHR 2020 Task Force on the Status and Future Direction of EHRs. Journal of American Medical Informatics Association. 2015;22(5):1102-1110.

27. United States Core Data for Interoperability (USCDI). https://www.healthit.gov/isa/united-states-core-data-interoperability-uscdi. Accessed 16 Oct 2020.

28. Watkins M, Viernes B, Nguyen V, et al. Translating social determinants of health into standardized clinical entities. Studies in Health Technology and Informatics. 2020;270:474-478.

29. Using Z codes: the Social Determinants of Health (SDOH). [infographic]. 2021; https://www.cms.gov/files/document/zcodes-infog raphic.pdf. Accessed 11 May 2021.

30. HL7 Gravity project. Ann Arbor (MI): Health Level Seven International; [accessed 2020 Oct 23]. [6 p]. Available: http://www.hl7. org/gravity/.

31. Office of the National Coordinator for Health Information T. Interoperability Standards Advisory (ISA). https://www.healthit.gov/ isa/. Accessed 21 May 2021.

32. Office of the National Coordinator for Health Information T. 2015 edition final rule: data segmentation for privacy (DS4P). Washington (DC): U.S. Department of Health and Human Services.

33. Health Privacy Rule 42 CFR Part 2 is revised, modernizing care coordination for Americans seeking treatment for substance use disorders. 2020; https://www.samhsa.gov/newsroom/press-announcements/202007131115. Accessed 13 July 2020.

34. International HL. Welcome to FHIR ®. http://hl7.org/fhir/?utm_referrer=https\%3A\%2F\%2Fwww.hl7.org\%2F.

35. Office of the National Coordinator for Health Information T. Data segmentation of sensitive information. https://www.healthit.gov/ isa/data-segmentation-sensitive-information.

36. Office for Civil Rights H. Information related to mental and behavioral health, including opioid overdose. https://www.hhs.gov/ hipaa/for-professionals/special-topics/mental-health/index.html.

37. Office of the National Coordinator for Health Information T. $\S 170.315(b)(7)$ Data segmentation for privacy - send. https://www. healthit.gov/test-method/data-segmentation-privacy-send.

38. Segal MJ. Patient safety and digital health tools: what are providers and developers focusing on at the end of 2020? 2020; https:// blog.ecri.org/patient-safety-and-digital-health-tools-what-are-providers-and-developers-focusing-on-at-the-end-of-2020. Accessed 8 Dec 2020.

39. Manley MV, Graber AL. Concepts in critical care: coronary prevention program in a community hospital. Heart and Lung the Journall of Cardiopulmonary and Care. 1977;6(6):1045-1049.

40. Mehrotra A, Chernew M, Linetsky D, et al. The impact of COVID-19 on outpatient visits in 2020: visits remained stable, despite a late surge in cases. 2021; https://doi.org/10.26099/bvhf-e411.

41. CMS proposes new rules to address prior authorization and reduce burden on patients and providers. 2020; https://www.cms.gov/ newsroom/press-releases/cms-proposes-new-rules-address-prior-authorization-and-reduce-burden-patients-and-providers. Accessed 22 Dec 2020. 
42. HHS proposes modifications to the HIPAA privacy rule to empower patients, improve coordinated care, and reduce regulatory burdens. 2020; https://www.hhs.gov/about/news/2020/12/10/hhs-proposes-modifications-hipaa-privacy-rule-empower-patients-improve-coord inated-care-reduce-regulatory-burdens.html. Accessed 22 Dec 2020.

43. Using information technology to integrate behavioral health and primary care. Behavioral health roundtable. Summary report of findings. (Prepared by: RTI International Center for the Advancement of Health Information Technology). Washington (DC): Office of the National Coordinator for Health Information Technology; 2012.

44. Reppeto H, Tuning C, Olsen DH, et al. Triple aim: benefits of behavioral health providers in primary care. Journal of Health Psychology. 2021;26(2):205-213.

Publisher's Note Springer Nature remains neutral with regard to jurisdictional claims in published maps and institutional affiliations. 